\title{
Finite-Time Stability for a Class of Underactuated Systems Subject to Time-Varying Disturbance
}

\author{
Jie Wu, ${ }^{1}$ Dan Yang, ${ }^{1}$ Xinyi He, ${ }^{1}$ and Xiaodi Li $\mathbb{D}^{1,2}$ \\ ${ }^{1}$ School of Mathematics and Statistics, Shandong Normal University, Jinan 250014, China \\ ${ }^{2}$ Center for Control and Engineering Computation, Shandong Normal University, Jinan 250014, China \\ Correspondence should be addressed to Xiaodi Li; sodymath@163.com
}

Received 24 March 2020; Accepted 29 April 2020; Published 12 May 2020

Academic Editor: Sigurdur F. Hafstein

Copyright (C) 2020 Jie Wu et al. This is an open access article distributed under the Creative Commons Attribution License, which permits unrestricted use, distribution, and reproduction in any medium, provided the original work is properly cited.

Based on the classical finite-time stability theory, the problem of finite-time stability (FTS) for time-varying nonlinear systems is investigated in this paper. Several FTS theorems involving global form and local form are presented, and an estimate of the settling-time of such systems is obtained. As an application, we consider the problem of asymptotic stabilization of the Brockett integrator subject to time-varying disturbance. By the switched finite-time controller design methodology, we establish a sufficient condition to guarantee the relative asymptotic stability. For Brockett-like integrator subject to time-varying disturbance, we achieve better convergence performance. Examples and their simulations are given to demonstrate the applicability of the proposed results.

\section{Introduction}

Over the past decades, many researchers have focused on Lyapunov asymptotic or exponential stability of dynamical systems, see [1-7]. One of the typical features of the asymptotic or exponential stability is that the solution will tend to an equilibrium state as time tends to infinity. Thus, it is difficult to achieve fast transient and high-precision performances. Finite-time stability (FTS) served as a special case of asymptotic stability means that the system reaches an equilibrium state in finite time, which presents an efficient tool for many engineering problems. Lots of interesting results on FTS have been raised from theoretical and practical points of view. Haimo [8] introduced a definition of continuous finite-time differential equations as fast accurate controllers for dynamical systems. Bhat and Bernstein [9] proposed Lyapunov theorem on FTS of continuous autonomous systems. Bhat and Bernstein [10] achieved globally FTS of the double integrator. Yang et al. [11] concerned the stabilization of switched dynamical networks with logarithmic quantization couplings in finite time. Moulay and Perruquetti [12] studied the FTS for a class of continuous systems using Lyapunov function. Furthermore,
Moulay et al. [13] established some FTS theorems for timedelay systems based on Lyapunov functionals and the extension of Artsteins transformation. Polyakov and $\mathrm{Hu}$ et al. $[14,15]$ introduced the concept of fixed-time stability, which means that the system is globally finite-time stable and the settling-time is bounded by some positive constant for any initial values. However, most of existing results, such as [8-13], are based on the framework of time invariant. Recently, Haddad et al. [16] obtained a sufficient condition of FTS for nonlinear time-varying systems. However, it can only be applied to some special cases due to the strict restriction that the derivative of Lyapunov function is less than zero almost everywhere. Moulay and Perruquetti [17] presented the FTS conditions for nonautonomous continuous systems. However, it was simplified to the time-invariant form through the inequality of the Lyapunov function. Hence, more general methods should be established for nonlinear time-varying systems. To avoid confusion, it should be pointed out that the FTS considered in this paper is different from another FTS concept adopted in [18-20], which dealt with the finite-time boundedness.

As stated in Brockett's result [21], Brockrtt integrator (Nonholonomic integrator), covered in underactuated 
systems, was the first example of locally controllable nonlinear system which is not smoothly stabilizable and requires additional constraints. In order to overcome the abovementioned limitations, various methods have been proposed for the problem of asymptotic stabilization of the Brockett integrator [22-26]. Astolfi [22] addressed the problem of almost exponential stabilization with bounded control. Banavar and Sankaranarayanan [23] studied a switched finite-time controller design methodology. Rehman [24] dealt with steering control of nonholonomic systems with drift for the extended nonholonomic double integrator. Rehman and Ahmed [25] presented piecewise constant and state-dependent feedback control for the nonholonomic integrator. Chihchen [26] investigated the time-varying control for globally exponential stabilization of the Brockett integrator. However, the abovementioned works did not take disturbance into account and converged in infinite time domain. Therefore, based on the FTS theorem for time-varying systems, we will explore stability issues of the Brockett integrator subject to time-varying disturbance.

In this paper, we firstly address some essential stability definitions in Section 2. Then, in Section 3, based on the classical finite-time stability theorem [9], the FTS theorem for time-varying nonlinear systems is considered. We apply it to solve the problem of asymptotic stabilization of the Brockett integrator subject to time-varying disturbance, as shown in Section 4. By some examples, we verify the main results in Section 5. In Section 6, conclusions are be presented.

\section{Preliminaries}

Notations: let $\mathbb{R}$ denote the set of real numbers, $\mathbb{R}_{+}$the set of nonnegative numbers, $\mathbb{R}^{n}$ the $n$-dimensional real spaces equipped with the Euclidean norm $|\cdot|$, and $\mathbb{N}, \mathbb{D} \subset \mathbb{R}^{n}$ the subspaces of $\mathbb{R}^{n} . a \vee b$ and $a \wedge b$ are the maximum and minimum of $a$ and $b$, respectively. The notation $A^{T}$ denotes the transpose of $A$.

Consider the nonlinear system given by

$$
\left\{\begin{array}{l}
\dot{x}(t)=f(t, x(t)), \\
x(0)=x_{0}
\end{array}\right.
$$

where $t \geq 0, \quad x \in \mathbb{R}^{n}$ denotes the state vector, $f(\cdot): \mathbb{R}_{+} \times \mathbb{D} \longrightarrow \mathbb{R}^{n}$ is a continuous function with $f(t, 0)=$ 0 for all $t \geq 0$. We assume that $f(t, x)$ satisfies suitable conditions so the solution $x(t)=x\left(t, 0, x_{0}\right)$ with initial state $x_{0} \in \mathbb{D}$ uniquely exists in forward time for all initial conditions except possibly the origin (see [9]).

Definition 1 (see [9]). System (1) is said to be FTS if there exist a function $T: \mathbb{R}^{n} \longmapsto \mathbb{R}_{+}$and an open neighborhood $\mathbb{N} \subseteq \mathbb{D}$ such that the following statements hold:

(i) Finite-time convergence (FTC): for every $x_{0} \in \mathbb{N} /\{0\}$, $x(t) \in \mathbb{N} /\{0\}$ holds for all $t \in\left[0, T\left(x_{0}\right)\right)$ and $x(t) \longrightarrow 0$ as $t \longrightarrow T\left(x_{0}\right)^{-}$

(ii) Lyapunov stability (LS): for every $\varepsilon$ ball $B_{\varepsilon}$ around the origin, there exists a $\delta$ ball $B_{\delta}$ around the origin such that, for every $x_{0} \in B_{\delta} /\{0\}, x(t) \in B_{\varepsilon}$ for all $t \in\left[0, T\left(x_{0}\right)\right)$

Definition 2 (see [23]). For a given set $\mathcal{O}$ that contains the origin, system (1) is said to be relatively asymptotically stable (RAS) with respect to the set $\mathcal{O}$, if for any $\varepsilon>0$, there exists $\delta>0$ such that, for all $x_{0} \in \mathcal{O} \cap B_{\delta}, x(t) \in B_{\varepsilon}$ holds when $t \geq 0$; moreover, $x(t) \longrightarrow 0$ as $t \longrightarrow \infty$. $B_{\delta}$ and $B_{\varepsilon}$ are open balls around the origin of radius $\delta$ and $\varepsilon$, respectively.

\section{Main Results}

In this section, we present a generalization of the classical FTS theorem [9]. Given the following definition

$$
\dot{V}(t, x(t)) \triangleq \frac{\partial V}{\partial t}(t, x)+\frac{\partial V}{\partial x}(t, x) f(t, x(t)),
$$

for a continuously differentiable function $V(t, x): \mathbb{R}_{+} \times \mathbb{R}^{n} \longrightarrow \mathbb{R}_{+}$. With a slight stealing concept, $V(t, x): \mathbb{R}_{+} \times \mathbb{R}^{n} \longrightarrow \mathbb{R}_{+}$is said to be positive definite and radially unbounded if there exists a positive definite and radially unbounded continuous function $W(x): \mathbb{R}^{n} \longrightarrow \mathbb{R}_{+}$ such that $V(t, x) \geq W(x)$, for $t \geq 0, x \in \mathbb{R}^{n}$.

Theorem 1. Consider system (1), if there exists a positive definite, continuously differentiable function $V: \mathbb{R}_{+} \times \mathbb{R}^{n}$ $\longrightarrow \mathbb{R}_{+}$, an integrable function $c(t): \mathbb{R}_{+} \longmapsto \mathbb{R}_{+}$, and two real numbers $\alpha \in(0,1), \sigma>0$, such that the derivative of $V$ along the solution $x(t)=x\left(t, 0, x_{0}\right)$ of system (1) satisfies

$$
\dot{V}(t, x(t)) \leq-c(t) V^{\alpha}(t, x(t)), \quad \forall t \geq 0,
$$

where $c(t)$ satisfies

$$
\int_{0}^{+\infty} c(s) \mathrm{d} s \triangleq \beta \geq \frac{\sigma^{1-\alpha}}{1-\alpha} .
$$

Then, system (1) is locally FTS with respect to $x_{0}$ satisfying $V_{0}<\sigma$, where $V_{0} \triangleq V\left(0, x_{0}\right)$. The settling-time function $T: \mathbb{R}^{n} \longmapsto \mathbb{R}_{+}$, depending on the initial state $x_{0}$, is bounded by

$$
T\left(x_{0}\right) \leq \inf \left\{t>0: \int_{0}^{t} c(s) \mathrm{d} s=\frac{V_{0}{ }^{1-\alpha}}{1-\alpha}\right\} .
$$

Moreover, when $\beta=+\infty$ and $V$ is radially unbounded, system (1) is globally FTS.

Proof. For a given $x_{0} \in \mathbb{R}^{n} /\{0\}$, let $x(t)$ be the solution of system (1) through $\left(0, x_{0}\right)$. By transforming (3), we obtain

$$
\frac{\mathrm{d} V}{V^{\alpha}} \leq-c(t) \mathrm{d} t, \quad \forall t \geq 0 .
$$
it gives

Integrating both sides of the abovementioned inequality,

$$
\int_{V_{0}}^{V(t, x(t))} \frac{1}{V^{\alpha}} \mathrm{d} V \leq \int_{0}^{t}-c(s) \mathrm{d} s .
$$

Since $V$ is a positive definite function, we can obtain 


$$
0 \leq \frac{V^{1-\alpha}(t, x(t))}{1-\alpha} \leq \frac{V_{0}^{1-\alpha}}{1-\alpha}-\int_{0}^{t} c(s) \mathrm{d} s .
$$

When $\beta<+\infty$ and $V_{0}<\sigma$, it follows from (4) that there exists a time point $t^{*}>0$ satisfying $\int_{0}^{t^{*}} c(s) \mathrm{d} s<\sigma^{1-\alpha} /(1-\alpha)$, such that $V_{0}^{1-\alpha} /(1-\alpha)-\int_{0}^{t^{*}} c(s) \mathrm{d} s=0$. That is to say $V\left(t^{*}, x\left(t^{*}\right)\right)=0$, from which the settling time $T\left(x_{0}\right)$ can be estimated, namely, (5). When $t \geq T\left(x_{0}\right), \dot{V}(t, x(t)) \leq 0$, it follows from [27] that the solution of system (1) through $\left(T\left(x_{0}\right), 0\right)$ is unique in forward time. Therefore, $V(t, x(t)) \equiv 0$, for $t \geq T\left(x_{0}\right)$, which means system (1) is locally FTC with respect to $x_{0}$ satisfying $V_{0}<\sigma$. Noting from (3), it is easy to obtain that system (1) is LS. Combining these two aspects, system (1) is locally FTS with respect to $x_{0}$ satisfying $V_{0}<\sigma$.

When $\beta=+\infty$ and $V$ is radially unbounded, no matter what initial value $x_{0}$ it is, there always exists a time point $t^{* *}>0$, such that $V_{0}^{1-\alpha} /(1-\alpha)-\int_{0}^{t^{* *}} c(s) \mathrm{d} s=0$, that is, $V\left(t^{* *}, x\left(t^{* *}\right)\right)=0$. Through a similar procedure, we conclude that system (1) is globally FTS.

Remark 1. The classical FTS theorem mentioned in [9] has been widely used in many fields, such as finite-time synchronizion of complex networks, finite-time attitude stabilization for spacecraft, and terminal sliding mode method of nonlinear systems, see [10, 23]. However, these theoretical results are applied under the framework of time invariant, that is, $c(t) \equiv c>0$. When $\inf \{c(t): t \geq 0\}=0$, the classical FTS theorem is unapplicable. However, in Theorem 1, we can still achieve the FTS of system (1), as long as (3) and (4) hold. This assertion can be verified in Section 5 .

\section{Applications}

Consider a class of systems described by equations of the form

$$
\dot{x}=G(x) u,
$$

where $x \in \mathbb{R}^{n}, u \in \mathbb{R}^{m}, m<n$, and $G(x)$ is a matrix of proper dimension. It has received considerable attention for the asymptotic stabilization of such systems during the past several decades. The reason for such an interest lies in the fact that system (9) cannot be asymptotically stabilized by any continuous differentiable and state feedback control laws (see [22]). The Brockett integrator, as a special case of system (9), has plenty of theoretical results with time-invariant form. However, there are few theoretical results considering the form of time-varying systems. In this paper, we study the Brockett integrator subject to time-varying disturbance, that is,

$$
\left\{\begin{array}{l}
\dot{x}_{1}(t)=u_{1}, \\
\dot{x}_{2}(t)=u_{2}, \\
\dot{x}_{3}(t)=w_{1}(t) x_{1} u_{2}-w_{2}(t) x_{2} u_{1},
\end{array}\right.
$$

where $t \geq 0, x_{i}(t): \mathbb{R}_{+} \longmapsto \mathbb{R}, i=1,2,3$, denotes the state component, $u_{j}(t): \mathbb{R}_{+} \longmapsto \mathbb{R}, j=1,2$, denotes the control input, $w_{j}(t): \mathbb{R}_{+} \longmapsto \mathbb{R}, j=1,2$, is continuous function denoting the disturbance term. For any integrable function $\rho(t): \mathbb{R}_{+} \longmapsto \mathbb{R}$, we introduce a set $\mathscr{F}$ as follows:

$$
\mathscr{F}=\left\{\rho(t) \in \mathbb{R}: \int_{0}^{t} \rho(s) \mathrm{d} s \longrightarrow+\infty \text { as } t \longrightarrow+\infty\right\} .
$$

Assumption 1. There exists a continuous function $\eta(t): \mathbb{R}_{+} \longmapsto \mathbb{R}$ such that $\eta(t) w_{1}(t) \geq 0$, for $t \geq 0$, and the following inequality holds:

$$
\int_{0}^{+\infty} \eta(s) w_{1}(s) \mathrm{d} s=+\infty
$$

Assumption 2. There exist two functions $\rho_{1}(t), \rho_{2}(t) \in F$ such that

$$
w_{1}(t) \rho_{2}(t)=w_{2}(t) \rho_{1}(t)
$$

Theorem 2. Assume that Assumptions 1 and 2 hold, then the solution of system (10) converges to the origin and system (10) is RAS with respect to the set $\mathcal{O} \triangleq\left\{x=\left(x_{1}\right.\right.$, $\left.\left.x_{2}, x_{3}\right)^{T} \in R^{3}: x_{3}=0, x_{1} \neq 0\right\}$ under the control input:

$$
U=\left(\begin{array}{l}
u_{1} \\
u_{2}
\end{array}\right)=\left(\begin{array}{c}
-\rho_{1}(t) x_{1} \\
\frac{-\eta(t) x_{3}{ }^{1 / 3}}{x_{1}}-\rho_{2}(t) x_{2}
\end{array}\right) .
$$

Proof. We firstly show the attractivity of system (10). If $x_{1}(0) \neq 0$, then it follows from (14) that

$$
\dot{x}_{1}(t)=-\rho_{1}(t) x_{1},
$$

which implies

$$
x_{1}(t)=x_{1}(0) \exp \int_{0}^{t}-\rho_{1}(s) \mathrm{d} s .
$$

Note that $\rho_{1}(t) \in F$; then, it is easy to see that

$$
x_{1}(t) \longrightarrow 0 \text { as } t \longrightarrow+\infty,
$$

which shows that $x_{1}(t)$ asymptotically tends to zero.

In addition, the third state component of system (10) becomes $\dot{x}_{3}(t)=-\eta(t) w_{1}(t) x_{3}{ }^{1 / 3}$ with control input $u_{2}$, which gives that $x_{3}(t)$ is FTS combining (12) and Theorem 1. Moreover, the settling time $T$ can be estimated by (5). For the second state component of system (10), when $t>T$, it holds $x_{3}{ }^{1 / 3} / x_{1} \equiv 0$; then, by (13), the derivative of $x_{2}$ becomes $-\rho_{2}(t) x_{2}$. Similar to the above argument, we could conclude that the origin of $x_{2}(t)$ asymptotically tends to zero. For the case that $x_{1}(0)=0$, we can apply any open loop control to steer the system to a nonzero value of $x_{1}$. This completes the proof of attractivity.

Secondly, we show the relative stability of system (10). When $t>T$, system (10) is transformed as 


$$
\begin{aligned}
& \dot{x}_{1}(t)=-\rho_{1}(t) x_{1}, \\
& \dot{x}_{2}(t)=-\rho_{2}(t) x_{2}, \\
& x_{3}(t)=0,
\end{aligned}
$$

which implies that $x(t)$ enters the set $\mathcal{O}$ from this moment, and $x_{1}(t)$ and $x_{2}(t)$ will converge to origin. Therefore, when $x(0) \in \mathcal{O}, x_{3}(t) \equiv 0 x_{1}(t), x_{2}(t) \longrightarrow 0$ as $t \longrightarrow \infty$, then one can obtain that system (10) is RAS with respect to the set $\mathcal{O}$. This completes the proof.

Remark 2. Theorem 2 provides a method for the problem of asymptotic stabilization of the Brockett integrator subject to time-varying disturbance. A relatively asymptotical stabilization is achieved by weakening the stability conditions. Besides, in the process of designing controller $U$, we apply a switched finite-time controller design methodology. In fact, it is also a discontinuous control. Worth mentioning that it is of vital importance to design functions $\eta(t), \rho_{1}(t)$, and $\rho_{2}(t)$, such that the FTS of $x_{3}(t)$ is achieved directly. In Section 5, we will show an example to illustrate our conclusion.

Corollary 1. Under conditions in Theorem 1, when $w_{1}(t)=w_{2}(t)$, we just need $\rho_{1}(t)=\rho_{2}(t)=p$, where $p$ is a positive constant. Then, the solution of system (10) converges to the origin and system (10) is RAS with respect to the set $\mathcal{O} \triangleq\left\{x=\left(x_{1}, x_{2}, x_{3}\right)^{T} \in R^{3}: x_{3}=0, x_{1} \neq 0\right\}$ under the control input:

$$
U=\left(\begin{array}{c}
u_{1} \\
u_{2}
\end{array}\right)=\left(\begin{array}{c}
-p x_{1} \\
\frac{-\eta(t) x_{3}^{1 / 3}}{x_{1}}-p x_{2}
\end{array}\right) .
$$

Remark 3. It should be pointed out that, in Theorem 2, the selection of set $\mathcal{O}$ and control input $U$ is not unique. In Theorem 2, we only get the FTS of one state component. In order to achieve better performance, we can further consider multiple components to achieve the FTS for the Brockettlike integrator, which is the result we will give in Theorem 3 .

Consider the following Brockett-like integrator subject to time-varying disturbance:

$$
\left\{\begin{array}{l}
\dot{x}_{1}(t)=u_{1}, \\
\dot{x}_{2}(t)=u_{2}, \\
\dot{x}_{3}(t)=w_{1}(t) x_{1}{ }^{k} u_{2}-w_{2}(t) x_{2} u_{1},
\end{array}\right.
$$

where $t \geq 0, x_{i}(t): \mathbb{R}_{+} \longmapsto \mathbb{R}, i=1,2,3$, denotes the state component, $u_{j}(t): \mathbb{R}_{+} \longmapsto \mathbb{R}, j=1,2$, denotes the control input, $w_{j}(t): \mathbb{R}_{+} \longmapsto \mathbb{R}, j=1,2$, is continuous function denoting the disturbance term, $k=a / b, a<b$, and $a$ and $b$ are positive odd numbers.

Assumption 3. There exists a continuous function $\mu(t): \mathbb{R}_{+} \longmapsto \mathbb{R}$ such that $\mu(t) w_{1}(t) \geq 0$, for $t \geq 0$, and the following inequality holds:

$$
\int_{0}^{+\infty} \mu(s) w_{1}(s) \mathrm{d} s=+\infty
$$

Assumption 4. There exist two functions $\varphi_{1}(t), \varphi_{2}(t) \in \mathscr{F}$ such that

$$
w_{1}(t) \varphi_{2}(t)=w_{2}(t) \varphi_{1}(t)
$$

Assumption 5. There exist two functions $T\left(x_{1}\right)=\inf$ $\left\{t>0, x_{1} \neq 0: \int_{0}^{t} \varphi_{1}(s) \mathrm{d} s=\left(x_{1}^{1-k} /(1-k)\right)\right\}, \quad T\left(x_{3}\right)=\inf$ $\left\{t>0: \int_{0}^{t} \mu(s) w_{1}(s) \mathrm{d} s=\left(\left(x_{3}{ }^{2 / 3}\right) / 2 / 3\right)\right\}$ such that the set $\mathscr{K} \triangleq\left\{x=\left(x_{1}, x_{2}, x_{3}\right)^{T} \in R^{3}: T\left(x_{3}\right)<T\left(x_{1}\right)\right\}$ is not empty.

Theorem 3. Assume that Assumptions 3, 4, and 5 hold; then, the solution of system (20) converges to the origin, and system (20) is RAS with respect to the set $\mathscr{Z} \triangleq\left\{x=\left(x_{1}, x_{2}\right.\right.$, $\left.\left.x_{3}\right)^{T} \in R^{3}: \mathscr{K} \cup\left\{(0,0,0)^{T}\right\}\right\}$ under the control input:

$$
U=\left(\begin{array}{c}
u_{1} \\
u_{2}
\end{array}\right)=\left(\begin{array}{c}
-\varphi_{1}(t) x_{1}{ }^{k} \\
\frac{-\mu(t) x_{3}{ }^{1 / 3}}{x_{1}^{k}}-\varphi_{2}(t) x_{2}
\end{array}\right) .
$$

Proof. If $x_{1}(0) \neq 0$, it follows from (23) that

$$
\left\{\begin{array}{l}
\dot{x}_{1}(t)=-\varphi_{1}(t) x_{1}^{k} \\
\dot{x}_{2}(t)=\frac{-\mu(t) x_{3}^{1 / 3}}{x_{1}^{k}}-\varphi_{2}(t) x_{2} \\
\dot{x}_{3}(t)=-\mu(t) w_{1}(t) x_{3}^{1 / 3}
\end{array}\right.
$$

About the first state component of system (20), we notice that $\varphi_{1}(t) \in \mathscr{F}$ and $k=a / b, a<b$, and $a$ and $b$ are positive odd numbers, so obtained from Theorem 1, the first state component of system (20) is FTS. Meanwhile, combining (21), (22), and Theorem 1, the third state components of system (20) is FTS. Hence, for initial value $x_{1}(0)$ and $x_{3}(0)$, the settling time can be established, that is, $T\left(x_{1}(0)\right)$ and $T\left(x_{3}(0)\right)$. Under the premise of Assumption 5, when $t>T\left(x_{3}(0)\right)$, system (20) is transformed as

$$
\begin{aligned}
& \dot{x}_{1}(t)=-\varphi_{1}(t) x_{1}{ }^{k}, \\
& \dot{x}_{2}(t)=-\varphi_{2}(t) x_{2}, \\
& x_{3}(t)=0 .
\end{aligned}
$$

Similar to the proof process of Theorem 2, we can obtain that system (20) is $R A S$ with respect to the set $\mathscr{Z}$. For the case that $x_{1}(0)=0$, we can apply any open loop control to steer the system to a nonzero value of $x_{1}$. This completes the proof.

\section{Numerical Simulations}

In this section, we will demonstrate the effectiveness of the proposed results for the above applications through the following simulation examples. 
Example 1. Consider the following 2D time-varying system:

$$
\left\{\begin{array}{l}
\dot{x}_{1}(t)=(\sin t-1) x_{1}{ }^{1 / 3}+\sin t x_{2}{ }^{2} x_{1}, \\
\dot{x}_{2}(t)=(\cos t-1) x_{2}{ }^{1 / 3}-x_{1}{ }^{2} x_{2}
\end{array}\right.
$$

where $t \geq 0$. Consider the Lyapunov function $V(x)=x_{1}{ }^{2}+x_{2}{ }^{2}$, and the derivative along the trajectory of the system (26) is

$$
\begin{aligned}
\dot{V}(t)= & 2 x_{1}\left((\sin t-1) x_{1}{ }^{1 / 3}+\sin t x_{2}{ }^{2} x_{1}\right) \\
& +2 x_{2}\left((\cos t-1) x_{2}{ }^{1 / 3}-x_{1}{ }^{2} x_{2}\right) \\
= & 2(\sin t-1) x_{1}{ }^{4 / 3}+2(\cos t-1) x_{2}{ }^{4 / 3} \\
& +2(\sin t-1) x_{1}{ }^{2} x_{2}{ }^{2} \\
\leq & 2(\sin t-1) x_{1}{ }^{4 / 3}+2(\cos t-1) x_{2}{ }^{4 / 3} \\
\leq & 2((\sin t-1) \vee(\cos t-1))\left(x_{1}{ }^{4 / 3}+x_{2}{ }^{4 / 3}\right) \\
\leq & -c(t) V^{2 / 3}(t),
\end{aligned}
$$

where $c(t)=2-2(\sin t \vee \cos t)$ and $c(t) \geq 0$ on $\mathbb{R}_{+}$. According to Lyapunov's stability theory in [28], system (26) is $L S$. It can be calculated that the lower bound of $c(t)$ is 0, so the classical FTS theorem in [9] is unapplicable. On the contrary, note that $c(t)$ satisfies (4) with $\beta=+\infty$, and it follows from Theorem 1 that system (26) is globally FTS and the settling time can be estimated as $T\left(x_{0}\right) \leq 4.51$. Figure 1 illustrates the state trajectories of system (26).

Example 2. Consider system (10) with $w_{1}(t)=t / 20$, $w_{2}(t)=1-\cos (t / 2), \eta(t)=1, \rho_{1}(t)=t / 200$, and $\rho_{2}(t)=$ $1 / 10-\cos (t / 2) / 10$. It is obvious that $w_{1}(t), w_{2}(t) \in \mathscr{F}, \eta(t)$ is a continuous function, and $w_{1}(t) \rho_{2}(t)=w_{2}(t) \rho_{1}(t)$. Hence, we can calculate that $\int_{0}^{+\infty} \eta(s) w_{1}(s) \mathrm{d} s=+\infty$. It follows from Theorem 2 that system (10) is RAS with respect to the set $\mathcal{O} \triangleq\left\{x=\left(x_{1}, x_{2}, x_{3}\right)^{T} \in \mathbb{R}^{3}: x_{3}=0, x_{1} \neq 0\right\}$, and the control input could be described by

$$
U=\left(\begin{array}{c}
u_{1} \\
u_{2}
\end{array}\right)=\left(\begin{array}{c}
-\left(\frac{t}{200}\right) x_{1} \\
\frac{-x_{3}{ }^{1 / 3}}{x_{1}}-\left(\frac{1}{10}-\frac{\cos (t / 2)}{10}\right) x_{2}
\end{array}\right) .
$$

It is worth pointing out that the classic FTS theorem in [9] is not applicable. This is due to the existence of the disturbance term, and the control input will reach infinity within a certain time, which is impractical. The numerical simulations for system (10) are follows.

In Figure 2, it can be clearly seen that $x_{3}(t)$ firstly converges to zero, then $x_{1}(t)$ and $x_{3}(t)$ converge to zero. The control input $u_{1}$ and $u_{2}$ change over time and turn into 0 when $x(t)$ converges to zero. In Figure 3 , the state vector $x(t)$ enters the set $\mathcal{O}$ and finally reaches the origin in finitetime. All of these results are corresponding to the conclusion of Theorem 2, which illustrates the validity of the Theorem 2.

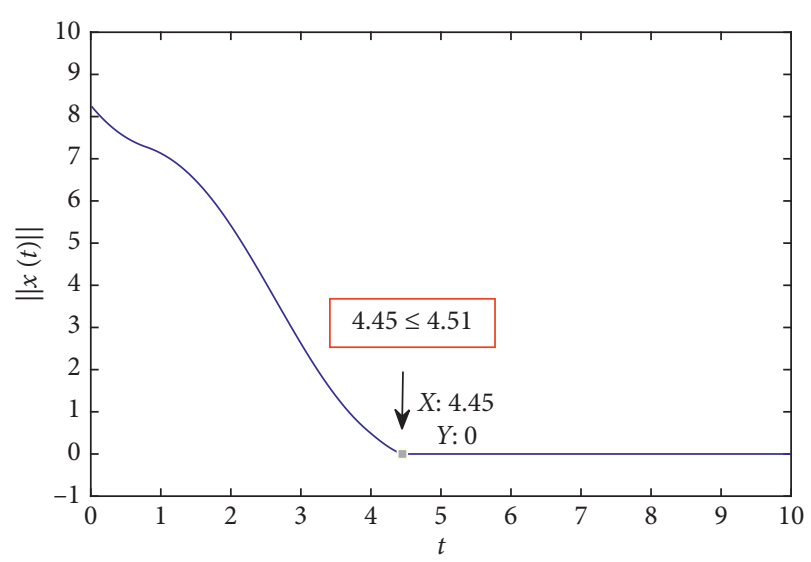

FIgURE 1: Simulation results of states for system (26).

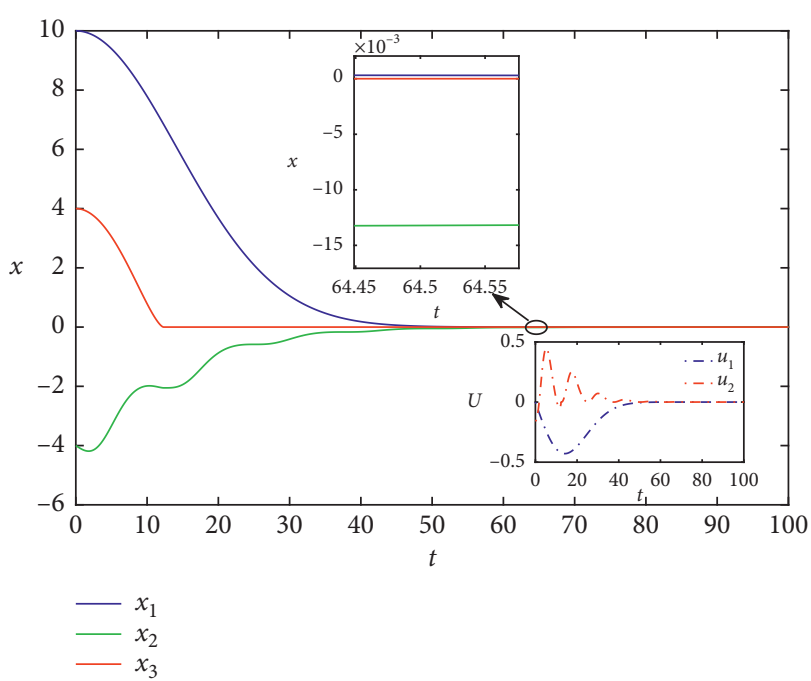

FIGURE 2: Simulation results of states and control inputs for system (10).

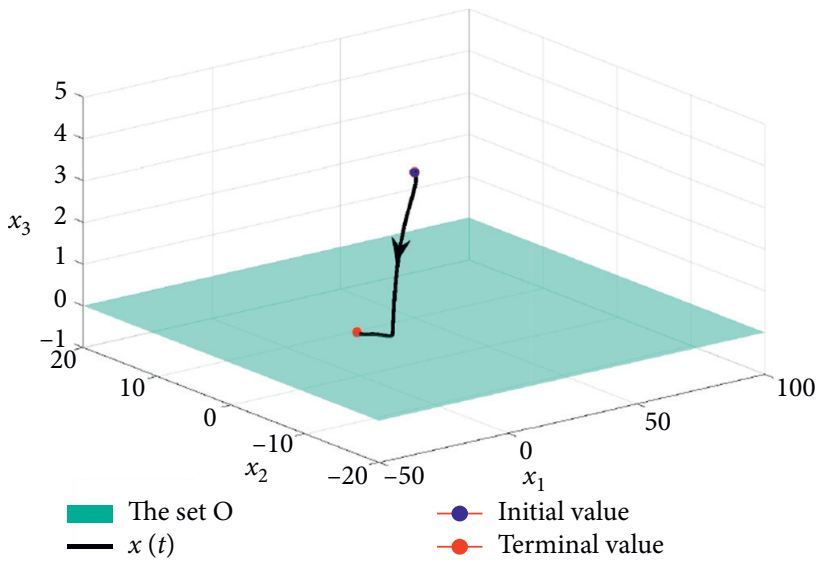

Figure 3: Simulation results of states for system (10).

Example 3. For convenience, the same parameters as in Example 2 are used. Consider system (2) with $w_{1}(t)=t / 20$, $w_{2}(t)=1-\cos (t / 2), \quad \quad \mu(t)=1, \quad \varphi_{1}(t)=t / 200$, 


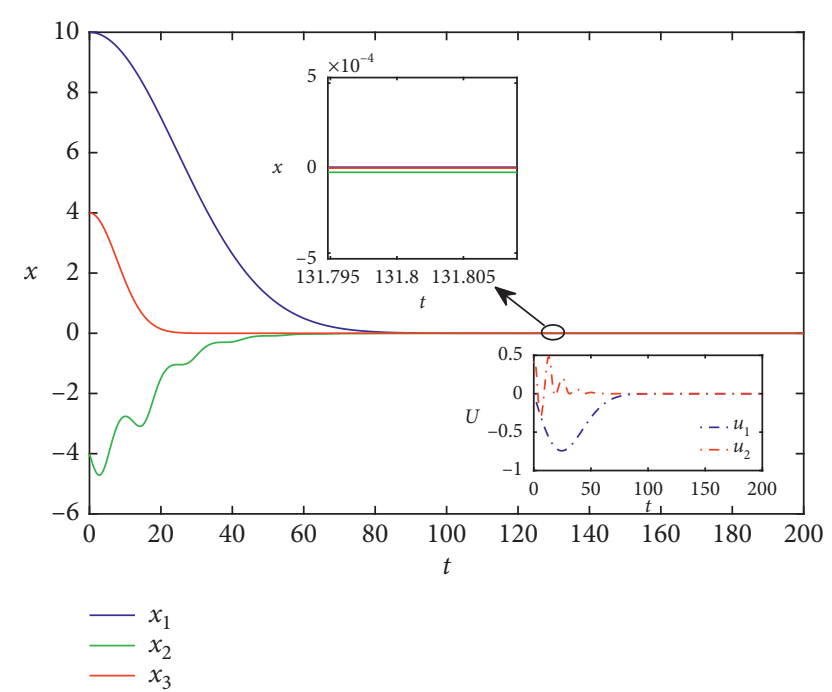

FIGURE 4: Simulation results of states and control inputs for system (20).

$\varphi_{2}(t)=1 / 10,-\cos (t / 2) / 10$, and $k=1 / 3$. It is not difficult to verify that the set $\mathscr{K}$ is not empty. Therefore, following from (21), (22), and Theorem 3, system (20) is RAS with respect to the set $\mathscr{Z}$ and the control input could be described by

$$
U=\left(\begin{array}{c}
u_{1} \\
u_{2}
\end{array}\right)=\left(\begin{array}{c}
-\left(\frac{t}{200}\right) x_{1}^{1 / 3} \\
\frac{-x_{3}^{1 / 3}}{x_{1}^{1 / 3}}-\left(\frac{1}{10}-\frac{\cos (t / 2)}{10}\right) x_{2}
\end{array}\right) .
$$

It can be seen from Figure 4 that the first and third state components of the system (20) is FTS, and the second state component of system (20) asymptotically tends to zero, which shows the validity of Theorem 3 .

\section{Conclusion}

The problem of FTS for time-varying nonlinear systems is investigated in this paper, where the results of global FTS and local FTS are proposed, respectively. As an application, the stabilization problem of the Brockett integrator subjecting to time-varying disturbance is studied. By the switched finite-time controller design methodology, we achieve the RAS for the Brockett integrator subject to timevarying disturbance. Further research topics would be considered to extend the main results of this paper to other more complex problems, such as the finite-time stabilization for wheeled mobile robot subject to time-varying disturbance and the FTS theorem for time-varying nonlinear systems in the sense of Lyapunov.

\section{Data Availability}

No data were used to support this study.

\section{Conflicts of Interest}

The author declares that there are no conflicts of interest.

\section{Acknowledgments}

This work was supported by the National Natural Science Foundation of China (61673247) and Research Fund for Distinguished Young Scholars and Excellent Young Scholars of Shandong Province (JQ201719).

\section{References}

[1] B. Zhou, "On asymptotic stability of linear time-varying systems," Automatica, vol. 68, pp. 266-276, 2016.

[2] J. Suo and J. Sun, "Asymptotic stability of differential systems with impulsive effects suffered by logic choice," Automatica, vol. 51, pp. 302-307, 2015.

[3] X. Li and J. Wu, "Stability of nonlinear differential systems with state-dependent delayed impulses," Automatica, vol. 64, pp. 63-69, 2016.

[4] C. Aouiti, I. Ben Gharbia, J. Cao, M. Salah M'hamdi, and A. Alsaedi, "Existence and global exponential stability of pseudo almost periodic solution for neutral delay BAM neural networks with time-varying delay in leakage terms," Chaos, Solitons \& Fractals, vol. 107, pp. 111-127, 2018.

[5] R. Rakkiyappan, G. Velmurugan, and J. Cao, "Stability analysis of fractional-order complex-valued neural networks with time delays," Chaos, Solitons \& Fractals, vol. 78, pp. 297-316, 2015.

[6] Y. Li and X. Meng, "Existence and global exponential stability of pseudo almost periodic solutions for neutral type quaternion-valued neural networks with delays in the leakage term on time scales," Complexity, vol. 2017, Article ID 9878369, 15 pages, 2017.

[7] R. Saravanakumar, M. S. Ali, C. K. Ahn, H. R. Karimi, and P. Shi, "Stability of Markovian jump generalized neural networks with interval time-varying delays," IEEE Transactions on Neural Networks and Learning Systems, vol. 28, no. 8, pp. 1840-1850, 2016.

[8] V. T. Haimo, "Finite time controllers," SIAM Journal on Control and Optimization, vol. 24, no. 4, pp. 760-770, 1986.

[9] S. P. Bhat and D. S. Bernstein, "Finite-time stability of continuous autonomous systems," SIAM Journal on Control and Optimization, vol. 38, no. 3, pp. 751-766, 2000.

[10] S. P. Bhat and D. S. Bernstein, "Continuous finite-time stabilization of the translational and rotational double integrators," IEEE Transactions on Automatic Control, vol. 43, no. 5, pp. 678-682, 1998.

[11] X. Yang, J. Cao, C. Xu, and J. Feng, "Finite-time stabilization of switched dynamical networks with quantized couplings via quantized controller," Science China Technological Sciences, vol. 61, no. 2, pp. 299-308, 2018.

[12] E. Moulay and W. Perruquetti, "Finite time stability and stabilization of a class of continuous systems," Journal of Mathematical Analysis and Applications, vol. 323, no. 2, pp. 1430-1443, 2006.

[13] E. Moulay, M. Dambrine, N. Yeganefar, and W. Perruquetti, "Finite-time stability and stabilization of time-delay systems," Systems \& Control Letters, vol. 57, no. 7, pp. 561-566, 2008.

[14] A. Polyakov, "Nonlinear feedback design for fixed-time stabilization of linear control systems," IEEE Transactions on Automatic Control, vol. 57, no. 8, pp. 2106-2110, 2012.

[15] J. Hu, G. Sui, X. Lv, and X. Li, "Fixed-time control of delayed neural networks with impulsive perturbations," Nonlinear Analysis: Modelling and Control, vol. 23, no. 6, pp. 904-920, 2018. 
[16] W. M. Haddad, S. G. Nersesov, and L. Du, "Finite-time stability for time-varying nonlinear dynamical systems," in Proceedings of the American Control Conference, IEEE, Seattle, WC, USA, pp. 4135-4139, 2008.

[17] E. Moulay and W. Perruquetti, "Finite time stability conditions for non-autonomous continuous systems," International Journal of Control, vol. 81, no. 5, pp. 797-803, 2008.

[18] F. Amato, R. Ambrosino, M. Ariola, C. Cosentino, and G. D. Tommasi, Finite-time Stability and Control, SpringerVerlag, London, UK, 2014.

[19] M. S. Ali, S. Saravanan, and J. Cao, "Finite-time boundedness, L2-gain analysis and control of Markovian jump switched neural networks with additive time-varying delays," Nonlinear Analysis: Hybrid Systems, vol. 23, pp. 27-43, 2017.

[20] X. Yang, X. Li, Q. Xi et al., "Review of stability and stabilization for impulsive delayed systems," Mathematical Biosciences \& Engineering, vol. 15, no. 6, pp. 1495-1515, 2018.

[21] R. W. Brockett, "Asymptotic stability and feedback stabilization," Differential Geometric Control Theory, vol. 27, no. 1, pp. 181-191, 1983.

[22] A. Astolfi, "Discontinuous control of the Brockett integrator," in Proceedings of the IEEE Conference on Decision \& Control, vol. 4, no. 1, pp. 49-63, IEEE, San Diego, CA, USA, December 1997.

[23] R. N. Banavar and V. Sankaranarayanan, Switched Finite Time Control of a Class of Underactuated Systems, Springer-Verlag, Berlin, Germany, 2006.

[24] F. Rehman, "Steering control of nonholonomic systems with drift: the extended nonholonomic double integrator example," Nonlinear Analysis-Theory Methods \& Applications, vol. 62, no. 8, pp. 1498-1515, 2005.

[25] F. Rehman and N. Ahmed, "Feedback stabilization of nonholonomic integrator (Brockett's system)," International Journal of Modelling and Simulation, vol. 26, no. 3, pp. 244-250, 2006.

[26] Y. Chihchen, "Time-varying control for exponential stabilisation of the Brockett integrator," IET Control Theory \& Applications, vol. 11, no. 12, pp. 1976-1982, 2017.

[27] T. Yoshizawa, Stability Theory by Liapunov's Second Method, The Mathematical Society of Japan, Tokyo, Japan, 1966.

[28] X. Liao, L. Wang, and P. Yu, Stability of Dynamical Systems, Elsevier, Amsterdam, Netherlands, 2007. 